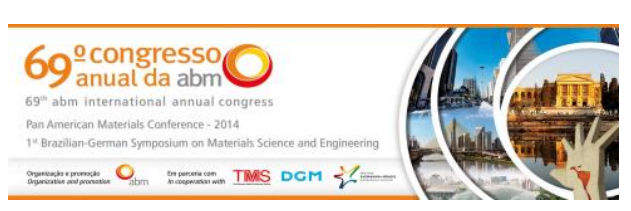

Tema: Metalurgia física e comportamento de materiais em temperaturas elevadas

\title{
INFLUÊNCIA DAS CARACTERÍSTICAS MICROESTRUTURAIS SOBRE AS PROPRIEDADES MAGNÉTICAS DO AÇO SILÍCIO DE GRÃO NÃO ORIENTADO 2\%Si*
}

\author{
José Rogério de Oliveira Júnior ${ }^{1}$ \\ Sebastião da Costa Paolinelli2 \\ Berenice Mendonça Gonzalez ${ }^{3}$ \\ Marcela do Valle Rodrigues ${ }^{4}$
}

\section{Resumo}

Neste trabalho foi determinada a influência do tamanho e da densidade de partículas sobre a perda magnética de um aço silício de grão não orientado (GNO) contendo $2 \% \mathrm{Si}$. Amostras provenientes de bobinas laminadas a frio após recozimento final, com dimensões de $305 \times 30 \times 0,54 \mathrm{~mm}$ e teores de carbono $32 \mathrm{ppm}$ e 46 ppm, foram submetidas a tratamentos de envelhecimento de 100 até $225^{\circ} \mathrm{C}$. A evolução da perda magnética durante o tratamento térmico de envelhecimento foi determinada interrompendo-se o tratamento em intervalos de tempo variados e realizando-se os testes magnéticos. Completado o ciclo de envelhecimento, todas as amostras foram avaliadas no microscópio eletrônico de varredura (MEV) para caracterização dos precipitados. Os resultados determinaram que o aumento da perda magnética ao longo do tempo, para uma dada temperatura, está associado à precipitação de carbonetos. O maior aumento da perda magnética encontrado correspondeu a uma distribuição de precipitados com tamanho médio entre 1,1 $\mu \mathrm{m}$ e 1,3 $\mu \mathrm{m}$ e a uma densidade de partículas de 0,025 partículas $/ \mu \mathrm{m}^{2}$.

Palavras-chave: Aço silício GNO; Envelhecimento magnético; Características microestruturais.

\section{INFLUENCE OF THE MICROSTRUCTURAL FEATURES ON THE CORE LOSS OF THE NON ORIENTED SILICON STEEL 2\%Si}

\section{Abstract}

In this work, the influence of the average particle size and the number of particles per unit area on the core loss of the $2 \% \mathrm{Si}$ non-oriented (NOG) silicon steel was determined. Cold rolled and annealed samples with $305 \times 30 \times 0,54 \mathrm{~mm}$ with $32 \mathrm{ppm}$ and $46 \mathrm{ppm}$ carbon contents, were subjected to ageing treatments from 100 to $225^{\circ} \mathrm{C}$. During the ageing treatment, in each sample, the cycle was interrupted at varied time intervals in order to determine the core loss. After that, the characterization of the precipitates was performed in the scanning electron microscope (SEM). The results have revealed that the increase on core loss along the time, at each temperature, is associated with iron carbides precipitation. The maximum increase on core loss for an average particle size between 1,1 $\mu \mathrm{m}$ and 1,3 $\mu \mathrm{m}$ and for particle density of 0,025 particles $/ \mu \mathrm{m}^{2}$.

Keywords: NOG silicon steel; Magnetic ageing; Microstructural features.

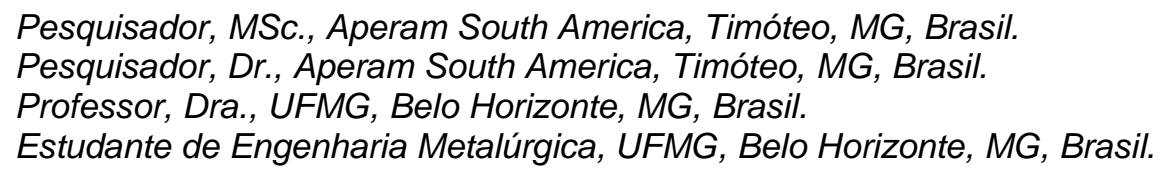

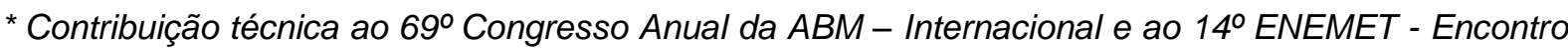
Nacional de Estudantes de Engenharia Metalúrgica, de Materiais e de Minas, 21 a 25 de julho de 2014, São Paulo, SP, Brasil.
} 


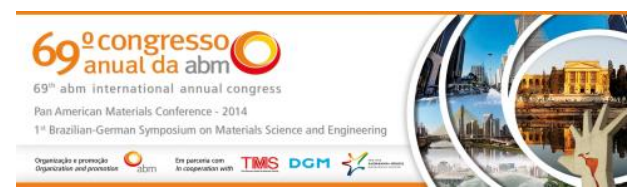

\section{INTRODUÇÃO}

Aços silício são materiais magneticamente macios empregados em núcleos de máquinas elétricas tais como transformadores e motores [1]. As principais características almejadas para esses materiais são: alta permeabilidade magnética e baixa perda magnética [2]. Os aços silício de grão não orientado (GNO) apresentam baixa anisotropia em relação às propriedades magnéticas [3,4] e são usualmente empregados em aparelhos onde o fluxo magnético muda de direção e sentido com o tempo, como em máquinas rotativas $[1,5]$.

A cada inversão de orientação do fluxo magnético, durante o funcionamento de uma máquina elétrica, há uma geração de calor associada à perda magnética do aço presente em seu núcleo. O consequente aumento de temperatura pode provocar a precipitação de uma fração do carbono em supersaturação, acarretando no aumento da força coerciva e consequentemente do componente histerético da perda magnética, deteriorando as propriedades magnéticas do material. Este fenômeno é conhecido como envelhecimento magnético [1,6-8].

$O$ carboneto $\varepsilon$ e a cementita são os dois principais tipos de carbonetos de ferro que podem precipitar em aços siliciosos e interferir nas suas propriedades magnéticas [1]. $O$ carboneto de transição $\varepsilon$ normalmente precipita em temperaturas da ordem de 100 a $250^{\circ} \mathrm{C}$, no interior dos grãos, provocando um aumento significativo na força coerciva. Já a precipitação da cementita, quando ocorre nos contornos de grão, é menos deletéria às propriedades magnéticas [1]. De maneira geral, o aumento da força coerciva depende das características magnéticas, da fração volumétrica, do estado de dispersão e do tamanho dos precipitados [9,10]. Precipitados com dimensões maiores que $0,1 \mu \mathrm{m}$ são considerados os mais deletérios às propriedades magnéticas [1,7]. Alguns autores reportaram que partículas com tamanho médio entre 1,2 e 1,8 $\mathrm{mm}$ provocaram maior acréscimo na coercividade em diversas ligas [11-13], sendo esta faixa de tamanhos considerada como crítica.

Neste trabalho, avaliou-se a variação percentual da perda magnética, denominada índice de envelhecimento magnético (IE), em amostras de aço silício GNO 2\%Si, com teores de carbono de 32 e 46ppm, submetidas a tratamentos de envelhecimento de 100 a $225^{\circ} \mathrm{C}$. Ao final de cada tratamento, foram determinadas as características dos precipitados formados, bem como a influência destas características sobre a variação da perda magnética.

\section{MATERIAIS E MÉTODOS}

Para realização dos experimentos, foram utilizadas chapas de aço silício GNO 2\%Si totalmente processado com dimensões $305 \times 30 \times 0,54 \mathrm{~mm}$ coletadas após recozimento final. As composições químicas destas encontram-se na Tabela 1.

Tabela 1. Composição química dos aços GNO 2\%Si (\% em peso)

\begin{tabular}{|c|c|c|c|c|c|c|c|}
\hline Aço & $\mathrm{C}$ & $\mathrm{Si}$ & $\mathrm{Mn}$ & $\mathrm{Al}$ & $\mathrm{P}$ & $\mathrm{S}$ & $\mathrm{N}$ \\
\hline 01 & 0,0032 & 2,0448 & 0,4121 & 0,0022 & 0,0185 & 0,0007 & 0,0034 \\
\hline 02 & 0,0046 & 2,0448 & 0,4121 & 0,0022 & 0,0185 & 0,0007 & 0,0034 \\
\hline
\end{tabular}

O tratamento térmico de envelhecimento foi realizado utilizando um forno tipo mufla dotado de resistência elétrica e ar como atmosfera. As amostras foram envelhecidas a $100 \pm 5^{\circ} \mathrm{C}, 150 \pm 5^{\circ} \mathrm{C}, 200 \pm 5^{\circ} \mathrm{C}$ e $225^{\circ} \mathrm{C} \pm 5^{\circ} \mathrm{C}$, com interrupção do ciclo para medição das propriedades magnéticas. $O$ final do tratamento foi alcançado

\footnotetext{
* Contribuição técnica ao $69^{\circ}$ Congresso Anual da ABM - Internacional e ao 14ํㅡㄹ ENEMET - Encontro Nacional de Estudantes de Engenharia Metalúrgica, de Materiais e de Minas, 21 a 25 de julho de 2014, São Paulo, SP, Brasil.
} 


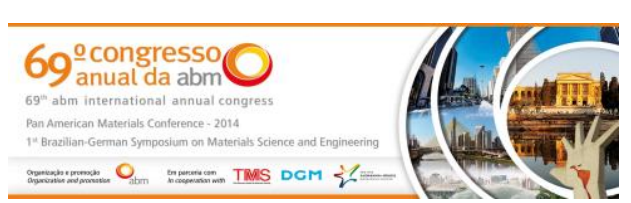

mantendo-se as amostras no forno até o tempo no qual a perda magnética não apresentasse mais variações significativas. Após este tempo de tratamento, foi realizada uma análise metalográfica no MEV para determinação dos parâmetros microestruturais.

As medições das propriedades magnéticas foram realizadas nas dimensões originais das amostras $(305 \times 30 \times 0,54 \mathrm{~mm})$, utilizando o acessório chapa única do medidor de propriedades magnéticas Brockhauss MPG100D, na frequência de $50 \mathrm{~Hz}$ e indução 1,0T. A variação percentual da perda magnética (IE), após cada tratamento de envelhecimento, foi determinada utilizando a seguinte relação $[7,8]$ (Equação 1):

$$
I E=\left(\frac{P_{T}^{E}-P_{T}}{P_{T}}\right)
$$

Onde $P_{T}^{E}$ é a perda magnética total do aço envelhecido e $P_{T}$ a perda magnética total do aço antes do envelhecimento.

As amostras envelhecidas foram analisadas no microscópio eletrônico de varredura (MEV) PHILIPS, modelo XL-30, utilizando o detetor de elétrons secundários SE (Secondary Electron), para determinação do número de partículas por micrômetro quadrado (densidade de partículas) e as dimensões das partículas. As análises quantitativas foram realizadas via análise de imagem através do software INCA Suite, sendo considerada a medida do maior comprimento da partícula para determinação do tamanho de cada precipitado. Já para determinação da densidade, foi quantificado o número de partículas com tamanho superior a 0,2 $\mu \mathrm{m}$ presente em uma área de $1685 \mu \mathrm{m}^{2}$. Todas as análises foram realizadas baseando-se em 16 campos por amostra.

\section{RESULTADOS}

A Figura 1 apresenta a variação do índice de envelhecimento em função do tempo de tratamento térmico para amostras do aço com 32ppm de carbono. Com exceção do tratamento a $100^{\circ} \mathrm{C}$, o índice de envelhecimento deste aço ultrapassou os 5\% de IE em intervalos de tempo da ordem de 10h. Para os tratamentos de envelhecimento entre 150 e $225^{\circ} \mathrm{C}$, Figura 1, notou-se que o máximo IE foi praticamente atingido com $100 \mathrm{~h}$ de tratamento e o maior IE ( $\approx 54 \%$ ) ocorreu a $180^{\circ} \mathrm{C}$. Embora lento, o aumento de perda magnética a $100^{\circ} \mathrm{C}$, ao longo do tempo, acarretou em um aumento de $20 \%$ no IE.

As amostras envelhecidas do aço com 32ppm de carbono revelaram uma precipitação intragranular, Figura 2, com uma densidade média de partículas consideravelmente elevada, principalmente nas condições de tratamento $100^{\circ} \mathrm{C} / 3100 \mathrm{~h}$ (a) e $150^{\circ} \mathrm{C} / 1100 \mathrm{~h}$ (b). A $100^{\circ} \mathrm{C}$, Figura 2 a), observou-se uma precipitação bastante fina de partículas de segunda fase, com cerca de $98 \%$ dos precipitados com tamanhos inferiores a $0,6 \mu \mathrm{m}$, conforme mostra a Tabela 2, não permitindo inferir sobre a forma dos precipitados. Apesar de certa heterogeneidade quanto ao tamanho dos precipitados após tratamento a 150 e $180^{\circ} \mathrm{C}$, Figura 2 b) e c), notou-se que boa parte dos precipitados apresentava formato de agulha $e$ tamanho de partícula inferior a $2 \mu \mathrm{m}$ (Tabela 2).

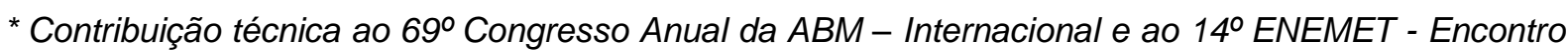
Nacional de Estudantes de Engenharia Metalúrgica, de Materiais e de Minas, 21 a 25 de julho de 2014, São Paulo, SP, Brasil.
} 


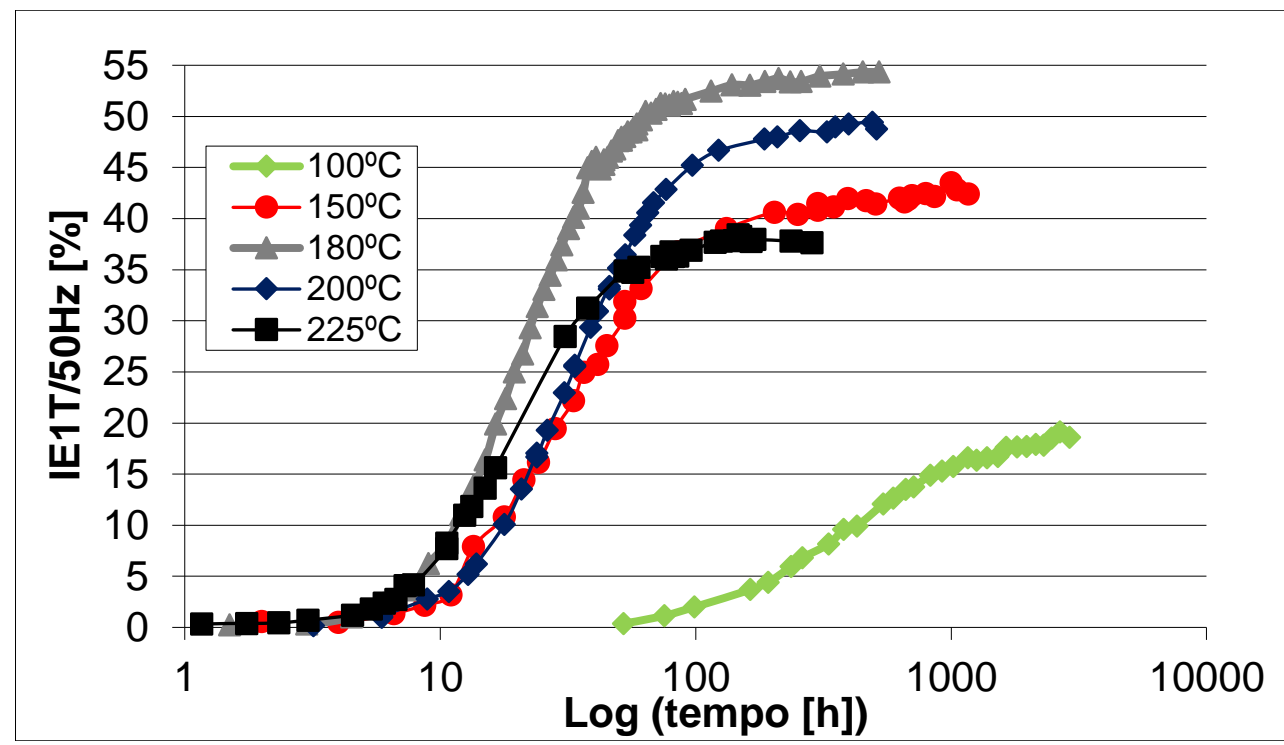

Figura 1. Curvas de envelhecimento magnético para o aço 1 (C:32ppm) a 100-225ํㅡ.
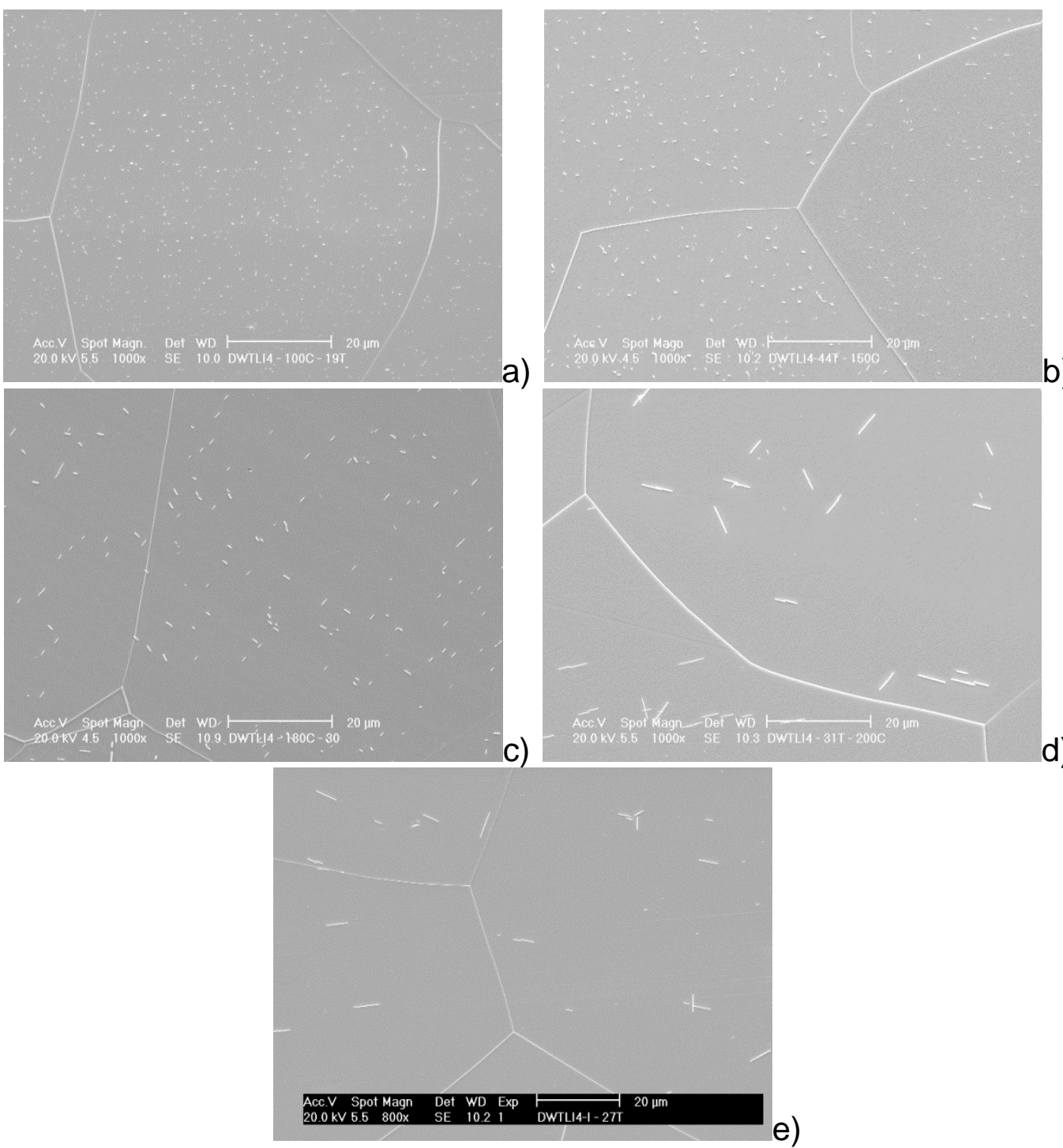

d)

e)

Figura 2. Micrografias, via MEV, de amostras do aço 1 (C:32ppm), após ensaio de envelhecimento. a) C:33ppm $100^{\circ} \mathrm{C} / 3100 \mathrm{~h} ;$ b)C:33ppm $150^{\circ} \mathrm{C} / 1100 \mathrm{~h} ;$ c) C:31ppm $180^{\circ} \mathrm{C} / 600 \mathrm{~h} ;$ d)C:33ppm $\left.200^{\circ} \mathrm{C} / 500 \mathrm{~h} ; \mathrm{e}\right) \mathrm{C}: 31 \mathrm{ppm} 225^{\circ} \mathrm{C} / 200 \mathrm{~h}$.

* Contribuição técnica ao 69 Congresso Anual da ABM - Internacional e ao 14ํㅡㄹ ENEMET - Encontro Nacional de Estudantes de Engenharia Metalúrgica, de Materiais e de Minas, 21 a 25 de julho de 2014, São Paulo, SP, Brasil. 


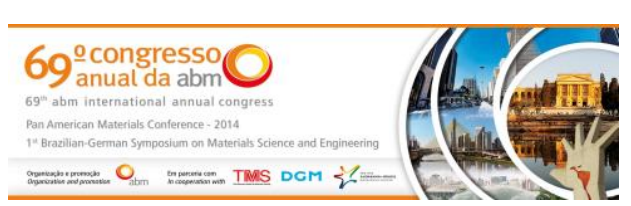

$\mathrm{O}$ tratamento a $180^{\circ} \mathrm{C}$, em relação à $150^{\circ} \mathrm{C}$, promoveu uma redução de 3 vezes na densidade média de partículas e um aumento no tamanho médio das partículas de 0,58 para $1,1 \mu \mathrm{m}$. A $180^{\circ} \mathrm{C}$, em torno de $50 \%$ dos precipitados apresentavam tamanhos entre 1,2 e $1,8 \mu \mathrm{m}$, conforme mostra a Tabela 2. A $200^{\circ} \mathrm{C}$ e $225^{\circ} \mathrm{C}$, Figura 2 d) e e), uma fração significativa dos precipitados observados possuíam forma de agulha e tamanho superior a $2 \mu \mathrm{m}$, Tabela 2 . O tratamento a $200^{\circ} \mathrm{C}$ provocou uma redução elevada na densidade média de partículas de aproximadamente 16 vezes, em relação ao tratamento a $180^{\circ} \mathrm{C}$, e, concomitantemente, elevou o tamanho médio para $3,33 \mu \mathrm{m}$.

Tabela 2. Distribuição de precipitados por faixa de tamanho, para cada temperatura aço 1(C:32ppm)

\begin{tabular}{|c|c|c|c|c|}
\hline \multirow{2}{*}{ Temperatura [을 } & \multicolumn{5}{|c|}{ Frequência relativa [\%] } \\
\cline { 2 - 5 } & $\leq 0,6 \mu \mathrm{m}$ & $0,61-1,1 \mu \mathrm{m}$ & $1,2-1,8 \mu \mathrm{m}$ & $\geq 2,0 \mu \mathrm{m}$ \\
\hline 100 & 98 & 1,5 & 0,5 & 0 \\
\hline 150 & 65 & 31 & 3 & 1 \\
\hline 180 & 14 & 32 & 50 & 4 \\
\hline 200 & 0 & 0 & 10 & 90 \\
\hline 225 & 0 & 12 & 12 & 76 \\
\hline
\end{tabular}

A variação do índice de envelhecimento em função do tempo de tratamento térmico para o aço com 46ppm de carbono é mostrada na Figura 3. O aumento do IE com o tempo para este aço mostrou-se extremamente sensível aos tratamentos térmicos entre 150 e $225^{\circ} \mathrm{C}$, presumidamente devido à maior supersaturação do carbono na matriz ferrítica. Com cerca de $4 \mathrm{~h}$ o IE, nesta faixa de temperatura, já era superior a $10 \%$. Para o tratamento a $10^{\circ} \mathrm{C}$, o aço com $46 \mathrm{ppm}$ de carbono atingiu um IE superior a $10 \%$ com um pouco mais de $100 \mathrm{~h}$. Já o aço com $32 \mathrm{ppm}$ de carbono, demorou cerca de $500 \mathrm{~h}$ para atingir os mesmos $10 \%$. Além disso, notou-se que com pouco mais de $20 \mathrm{~h}$ o aumento percentual de perda magnética em função do tempo, para 200 e $225^{\circ} \mathrm{C}$, já havia estabilizado. O máximo IE atingido para o aço com 46 ppm de carbono foi cerca de $80 \%$ e ocorreu a $200^{\circ} \mathrm{C}$ após $400 \mathrm{~h}$. Apenas no caso do aço com 32ppm de carbono percebeu-se a necessidade de um tratamento de envelhecimento em temperaturas intermediárias entre 150 e $200^{\circ} \mathrm{C}$ para se atingir $\mathrm{O}$ máximo IE da liga. As amostras do aço com 46ppm de carbono, submetidas ao tratamento na faixa $100-225^{\circ} \mathrm{C}$, apresentaram uma maior densidade média de partículas precipitadas no interior dos grãos, quando comparadas com o aço com 32ppm de carbono, Figura 4.

\footnotetext{
* Contribuição técnica ao $69^{\circ}$ Congresso Anual da ABM - Internacional e ao 14ํㅡㄹ ENEMET - Encontro Nacional de Estudantes de Engenharia Metalúrgica, de Materiais e de Minas, 21 a 25 de julho de 2014, São Paulo, SP, Brasil.
} 


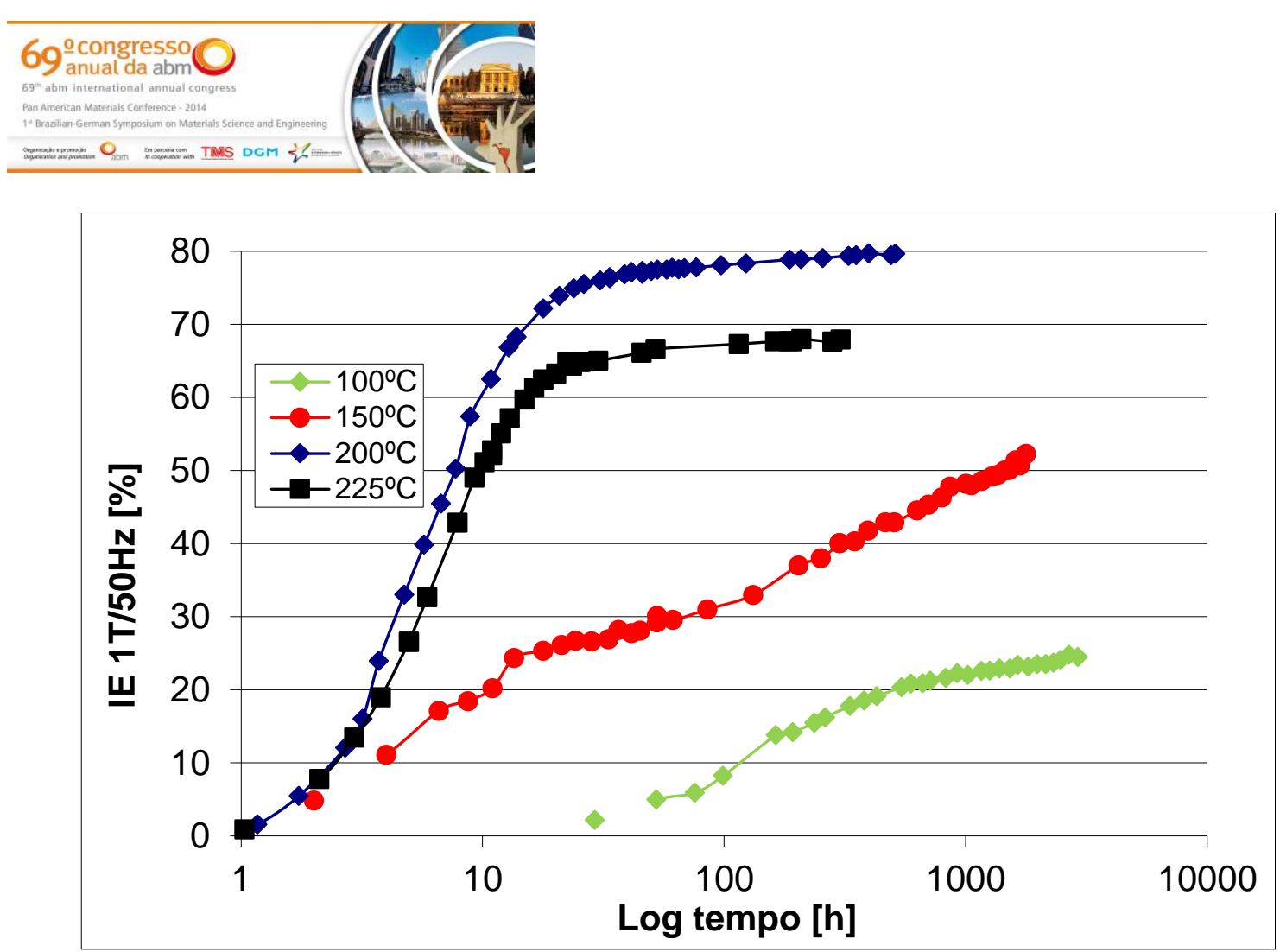

Figura 3. Curvas de envelhecimento magnético para o aço 2 (C:46ppm) a 100-225ํ․

Ao final do tratamento a $100^{\circ} \mathrm{C}$, Figura 4 a), praticamente todos os precipitados analisados apresentavam tamanhos inferiores a 0,6 $\mu \mathrm{m}$, Tabela 3 , o que dificultou a determinação da forma das partículas. A amostra tratada a $150^{\circ} \mathrm{C}$, Figura $4 \mathrm{~b}$ ), apresentou uma densa precipitação intragranular semelhante à observada a $100^{\circ} \mathrm{C}$. Entretanto, é possível notar a presença de outra distribuição de precipitados, no interior dos grãos, em forma de agulha, caracterizando uma típica distribuição bimodal. A $150^{\circ} \mathrm{C}$, em torno de $86 \%$ dos precipitados possuíam tamanhos inferiores a $0,6 \mu \mathrm{m}$ e $14 \%$ entre 0,61 e $1,8 \mu \mathrm{m}$, conforme mostra a Tabela 3 . A $200^{\circ} \mathrm{C}$ e $225^{\circ} \mathrm{C}$, Figura $4 \mathrm{c}$ ) e d), respectivamente, nota-se que boa parte dos precipitados apresenta forma de agulha. A $200^{\circ} \mathrm{C}$, cerca de $89 \%$ dos precipitados estavam entre 0,61 e $1,8 \mu \mathrm{m}$, Tabela 3, compreendendo uma faixa similar à obtida para o aço com 32ppm de carbono para $180^{\circ} \mathrm{C}$ (Tabela 2). Diferentemente do aço com 32ppm de carbono, o tratamento do aço com $46 \mathrm{ppm}$ de carbono a $225^{\circ} \mathrm{C}$ em relação à $200^{\circ} \mathrm{C}$, promoveu um aumento significativo no tamanho médio de partículas, levando o tamanho médio de partículas de 1,3 para $2,7 \mu \mathrm{m}$, respectivamente. Adicionalmente, a Tabela 3 mostra que o $80 \%$ dos precipitados, após o tratamento a $225^{\circ} \mathrm{C}$ do aço com $46 \mathrm{ppm}$ de carbono, possuíam tamanho superior a $2 \mu \mathrm{m}$.

Tabela 3. Distribuição de precipitados por faixa de tamanho, para cada temperatura aço 2(C:46ppm)

\begin{tabular}{|c|c|c|c|c|}
\hline \multirow{2}{*}{ Temperatura $\left[{ }^{\circ} \mathrm{C}\right]$} & \multicolumn{4}{|c|}{ Frequência relativa $[\%]$} \\
\cline { 2 - 5 } & $\leq 0,6 \mu \mathrm{m}$ & $0,61-1,1 \mu \mathrm{m}$ & $1,2-1,8 \mu \mathrm{m}$ & $\geq 2,0 \mu \mathrm{m}$ \\
\hline 100 & 98,6 & 1,2 & 0,2 & 0 \\
\hline 150 & 86 & 11 & 3 & 0 \\
\hline 200 & 0 & 18 & 71 & 11 \\
\hline 225 & 0 & 1 & 19 & 80 \\
\hline
\end{tabular}

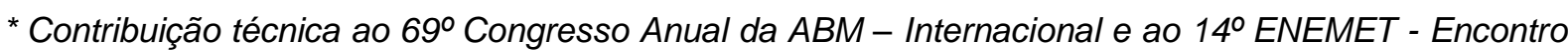
Nacional de Estudantes de Engenharia Metalúrgica, de Materiais e de Minas, 21 a 25 de julho de 2014, São Paulo, SP, Brasil.
} 

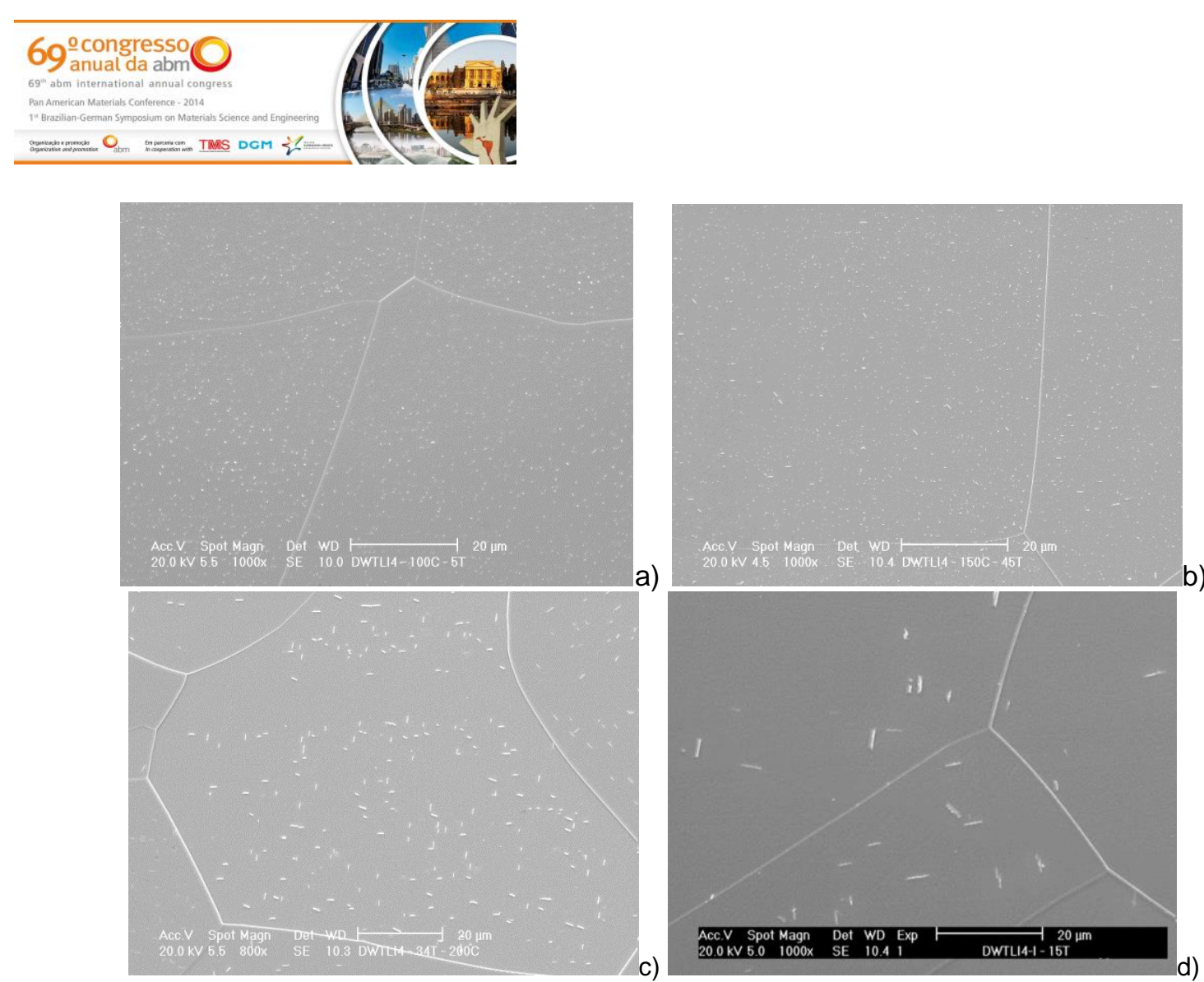

Figura 4. Micrografias, via MEV, de amostras do aço 2, após ensaio de envelhecimento. a)C:48ppm

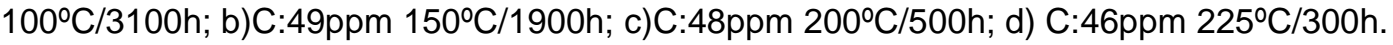

\section{DISCUSSÃO}

Embora 98\% dos precipitados da amostra com teor de carbono médio de 32ppm tratada a $100^{\circ} \mathrm{C}$, Figura 2 a), apresentassem tamanhos inferiores a 0,6 $4 \mathrm{~m}$, Tabela 2 , a elevada densidade de partículas foi a responsável pelo aumento de quase $20 \%$ na perda magnética deste aço, Figura 1. Ou seja, a variação da perda magnética, durante um tratamento de envelhecimento, assim como reportado por Leslie [14], depende do tamanho assim como da densidade das partículas. A $180^{\circ} \mathrm{C}$, uma distribuição de partículas com densidade 0,025 partículas $/ \mu m^{2}$, Figura $2 \mathrm{c}$ ), somada a presença de $50 \%$ dos precipitados na faixa considerada crítica $(1,2-1,8 \mu \mathrm{m})[12,13]$, Tabela 2, promoveram o maior aumento de perda magnética para esta liga. A presença de aproximadamente $10 \%$ dos precipitados na faixa crítica justificou o elevado IE alcançado pelos tratamentos a 200 e $225^{\circ} \mathrm{C}$ para as amostras do aço 1.

Eloot e colaboradores [15] mostraram que existe um aumento linear entre a perda magnética e o teor de carbono intersticial, a partir de 12ppm até aproximadamente $50 \mathrm{ppm}$. Dessa forma, o elevado teor de carbono presente nas amostras do aço com $46 \mathrm{ppm}$ de carbono, foi o responsável pelo aumento acentuado observado nos valores de perda magnética (Figura 3), principalmente para as temperaturas de 200 e $225^{\circ} \mathrm{C}$. A presença de $71 \%$ dos precipitados compreendidos pela faixa citada como crítica [12,13], Tabela 3, somada a uma densidade de aproximadamente 0,025 partículas $/ \mu \mathrm{m}^{2}$, Figura $4 \mathrm{c}$ ), foram os principais causadores de um aumento de $80 \%$ no IE para o tratamento a $200^{\circ} \mathrm{C}$, Figura 3 . Assim como mencionado anteriormente, o tratamento a $150^{\circ} \mathrm{C}$ para a amostra do aço 2 (C:46ppm), promoveu uma distribuição bimodal do tamanho de partículas. Uma análise mais detalhada da distribuição mostrada na Figura 4b mostrou a existência de partículas na ordem de $0,3 \mu \mathrm{m}$ e partículas com $1 \mu \mathrm{m}$. Adicionalmente, observou-se que a curva de envelhecimento apresentou um formato bem atípico em relação às outras curvas e

\footnotetext{
* Contribuição técnica ao 69ำ Congresso Anual da ABM - Internacional e ao 14ํㅡㄹ ENEMET - Encontro Nacional de Estudantes de Engenharia Metalúrgica, de Materiais e de Minas, 21 a 25 de julho de 2014, São Paulo, SP, Brasil.
} 
uma espécie de "degrau" com aproximadamente 10h de teste, conforme mostrado na Figura 3. Atribui-se a presença do "degrau", uma mudança no mecanismo de precipitação, presumidamente indicando o início do coalescimento de uma fração das partículas. Uma distribuição semelhante de precipitados também foi observada por Michal e Slane [11], durante o envelhecimento de uma liga Fe-2,3\%-0,7\%Si com 90 ppm de carbono a $150^{\circ} \mathrm{C}$. Foi identificado pelos autores, que a maior parte dos precipitados apresentava tamanhos da ordem de $0,12 \mu \mathrm{m}$, mas muitas partículas coalesceram e atingiram tamanhos da ordem de $0,5 \mu \mathrm{m}$, causando um aumento no IE da ordem de $22 \%$.

É interessante ainda notar que independente da temperatura de tratamento,

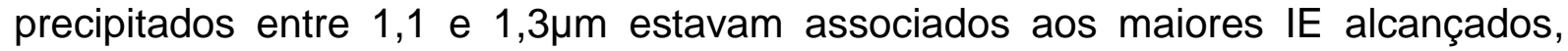
conforme ilustra a Figura 5. Valor este próximo à faixa crítica [12,13].

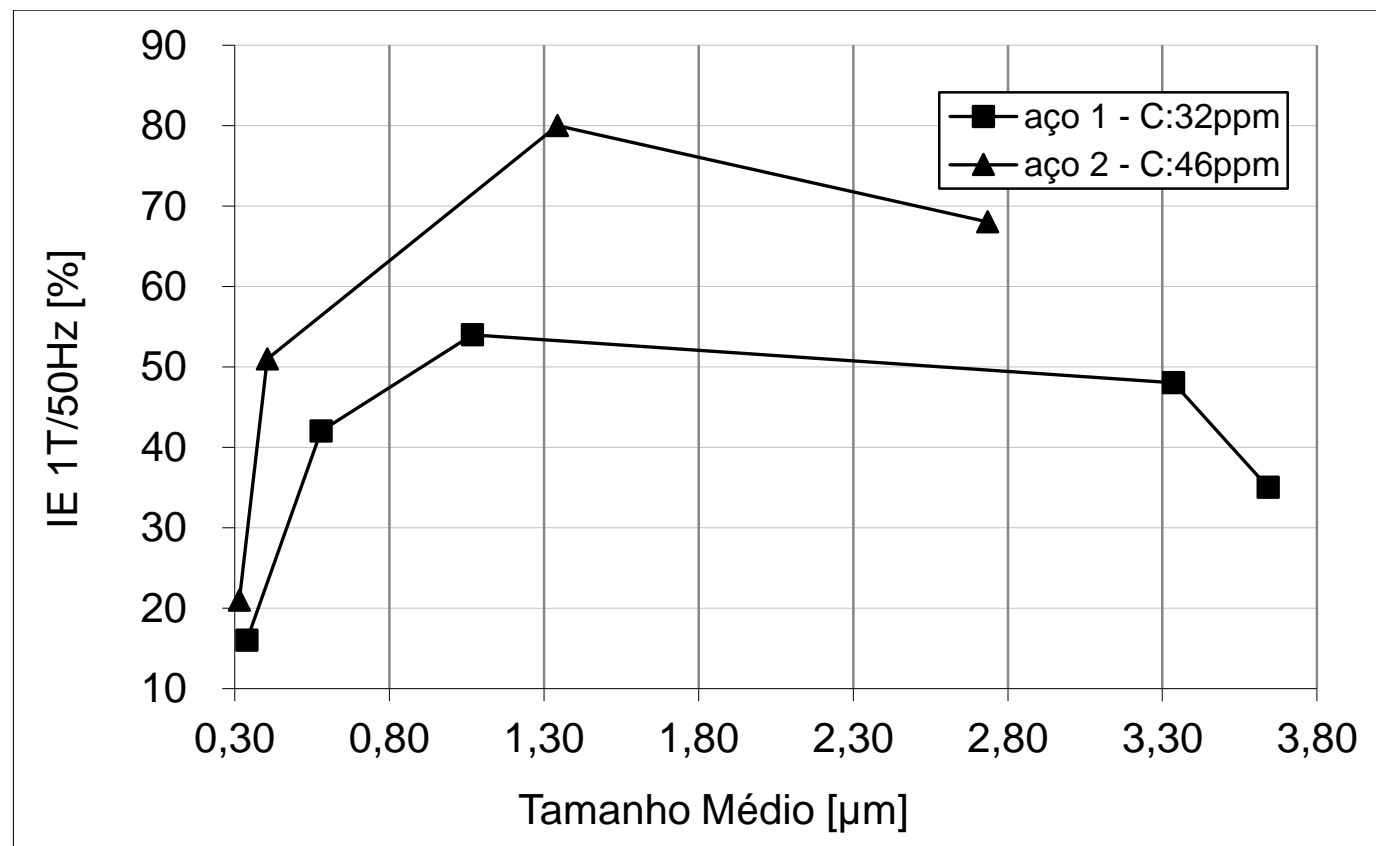

Figura 5. Efeito do tamanho médio de precipitados sobre o índice de envelhecimento magnético para os dois aços.

Semelhantemente, associado ao máximo de envelhecimento nos dois aços, observou-se também uma densidade crítica de partículas da ordem de 0,025partículas $/ \mu \mathrm{m}^{2}$, conforme mostra a Figura 6.

* Contribuição técnica ao 69 Congresso Anual da ABM - Internacional e ao 14ํㅡㄹ ENEMET - Encontro Nacional de Estudantes de Engenharia Metalúrgica, de Materiais e de Minas, 21 a 25 de julho de 2014, São Paulo, SP, Brasil. 


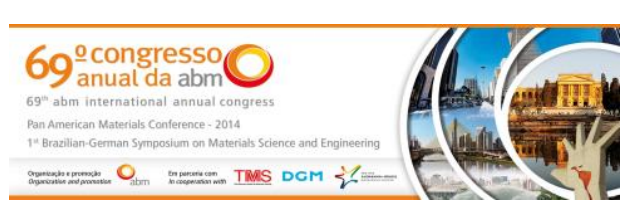

4 Takami KM. Process control and simulation of ferromagnetic strip in the power transformers and electrical machines applications. Malardalen University. Västerås, p.55. 2009 . (Ph.D. Thesis in Electrical Power Systems).

5 Paolinelli SC. Non-oriented silicon steels with improved texture. Proceedings 5th International Conference on Magnetism and Metallurgy. Ghent. 2012. p. 120-136.

6 Lindenmo M, Jenkins K. Precipitates in electrical steels. Journal of Magnetism and Magnetic Materials, 2008; 320: 2423-2429.

7 Marra KM. Cinética de Descarbonetação de um Aço GNO Semiprocessado e sua Influência nas Propriedades Magnéticas e na Susceptibilidade ao Envelhecimento Magnético. UFMG. Belo Horizonte, p. 283. 2002. (Tese de Doutorado em Engenharia Metalúrgica).

8 Negri GMR. Estudo de caso de envelhecimento magnético em aços elétricos de grão não orientado de baixa eficiência. UFSC. Florianópolis, p. 117. 2011. (Dissertação de Mestrado em Engenharia Elétrica).

9 Ray SK, Mohanty ON. On predicting the extent of magnetic aging in electrical steels. Journal of Magnetism and Magnetic Materials, 1989; 78: 255-262.

10 Ray SK, Mohanty ON, Mishra S. A study of the kinetics of magnetic aging in steels. IEEE Transactions on Magnetic, 1981; 17(6): 2881-2883.

11 Michal GM, Slane JA. The kinetics of carbide precipitation in silicon-aluminum steels. Metallurgical Transactions A, 1986; 17A: 1287-1294.

12 Leslie WC, Fisher M, Sen N. Morphology and Crystal Structure of Carbides Precipitated from Solid Solution in Alpha Iron. Acta Metallurgica, 1959; 7: 632-644.

13 Nacken M, Heller W. The Change in Coercivity during Aging of Unalloyed Mild Steel. Arch. Eisenhüttenw, 1960; 31: 153-160.

14 Leslie WC. The physical metallurgy of steels. Auckland: Mcgraw-Hill, 1981. p. 396.

15 Eloot K. et al. Evaluation of the susceptibility of an electrical steel to magnetic aging using new internal friction technique. Journal of Magnetism and Magnetic Materials, 1994; 133: 223-225.

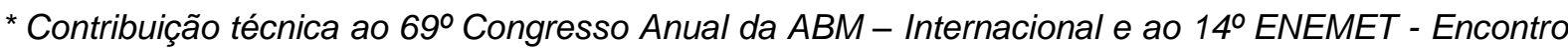
Nacional de Estudantes de Engenharia Metalúrgica, de Materiais e de Minas, 21 a 25 de julho de 2014, São Paulo, SP, Brasil. 